\title{
On the Spectral Efficiency of MIMO Two-way Multihop Networks with Co-existing Flows
}

\author{
Rindranirina Ramamonjison, Jonghyun Lee, Kei Sakaguchi, and Kiyomichi Araki \\ Tokyo Institute of Technology \\ 2-12-1 Ookayama, Meguro-ku, Tokyo, 152-8552 Japan \\ Email: $\{$ rindra $\mid$ hyun | sakaguchi $\mid$ araki\} @mobile.ee.titech.ac.jp
}

\begin{abstract}
In this paper, we investigate the spectral efficiency of MIMO two-way multihop networks when multiple flows co-exist and share the same channel. We assume that each multihop flow mitigates only its own intra-flow interference. More precisely, spatial array processing cancels adjacent node interference and a jointly optimal routing and power allocation strategy mitigates the weaker interference from far nodes. By assuming two types of topology, we study the effect of the inter-flow interference on the end-to-end capacity when the density of flows increases. For the linear topology, we show that multihop routing significantly improved the overall spectral efficiency in dense multihop networks; while single hop MIMO performed better at high SNR regime when there is no interfering flow nearby. However, the network was vulnerable to inter-flow interference in the parallel topology. Consequently, distributed admission control and resource allocation schemes, with coordination among the flows, will be of interest to further improve the spectral efficiency of the multihop networks.
\end{abstract}

\section{INTRODUCTION}

Wireless multihop networks will be a key component of future ubiquitous networks. In fact, they offer several benefits such as coverage extension, robustness to shadowing and ease of deployment. Thanks to their distributed architecture, multihop networks also enable a broad range of innovative applications such as industrial plant control or smart utility environments [1].

Although these applications are expected to require high data rate, current multihop networks suffer from a throughput degradation when the number of nodes increases [2]. This degradation is mainly due to the way spectrum is currently used to avoid interference. For instance, random MAC protocols with collision avoidance (e.g. CSMA/CA) leads to spectral inefficiency in multihop networks. It is also the case for orthogonal MAC which assigns a different channel to each link in one multihop flow.

Routing, which is the process of selecting a path to forward the packets between two end-nodes, has also an impact on the achievable throughput. It determines the intermediate relay nodes, their numbers and their locations. In contrast to mobile ad-hoc networks, we assume fixed relay nodes such as in backbone mesh networks.

Under the orthogonal MAC assumption, it was shown in [5], [6], [7] that multihop routing is advantageous in power-limited regime but less spectrally efficient than single hop routing due to the orthogonality loss. Furthermore, when simultaneous flows co-exist in the network, multihop routing with lower transmit power can increase the area spectral efficiency at the expense of the end-to-end throughput [7]. This fundamental trade-off makes the provision of high data rates for multiple flows even harder under an orthogonal MAC assumption.

Channel reuse, however, creates interference which also deteriorates the throughput. Unlike cellular networks, interference management in multihop networks is a greater challenge. When the same channel is simultaneously used by all links in a single flow, the resulting intra-flow interference can significantly reduce the end-to-end throughput. In addition, if multiple flows share the same channel, inter-flow interference also arises. Therefore, advanced spectrum sharing techniques and new interference management strategy are needed to improve the spectral efficiency of multihop networks.

To address these issues, we propose a novel network architecture, called MIMO two-way multihop network [3], in which all links of a single flow share the same spectrum channel. With multiple antennas at each node, spatial signal processing is performed to multiplex bidirectional streams in the multihop path and also to cancel all adjacent node interference. Furthermore, we mitigate the remaining interference from far nodes with a cross-layer approach by jointly optimizing the routing and power allocation.

Our objective is to investigate the spectral efficiency of the MIMO two-way multihop networks when co-existing flows are further allowed to share the same channel. To this end, we considered the linear and parallel flow topologies and analyzed the effect of the inter-flow interference on the end-toend capacity when the density of flows increases. We have not, however, addressed in this work the mitigation of inter-flow interference. Although we assumed that each flow mitigates only its own intra-flow interference, we show that multihop routing with optimal power allocation significantly improved the overall spectral efficiency in dense multihop networks, especially in the linear topology. Furthermore, single hop MIMO transmission provided higher throughput in high SNR regimes only when there is no interfering flow nearby. Finally, we observed that the network is more vulnerable to inter-flow interference in the parallel topology.

In Section II, we describe the MIMO two-way multihop network model and present the cross-layer approach for mitigating the intra-flow interference. In Section III, we analyze the effect of inter-flow interference on the spectral efficiency of the network with co-existing flows. 


\section{INTRA-FLOW INTERFERENCE MANAGEMENT}

\section{A. MIMO Two-way Multihop Network Model}

A MIMO two-way multihop network differs from conventional multihop networks in two important features: bidirectional link multiplexing and adjacent node interference cancellation [3].

First, MIMO spatial multiplexing enables the simultaneous transmission of forward and backward streams by each transmit (TX) node to its following and preceding receive (RX) nodes as described in Figure 1 and 2. Along the MIMO two-way multihop path, TX and RX nodes are then arranged alternatively at each time slot. However, at the next time slot, all TX nodes become RX nodes and vice versa. Consequently, two time slots are only required to achieve an end-to-end bidirectional transmission.

Second, all interference coming from adjacent node are spatially cancelled with MIMO techniques. This feature is very important since all links in the multihop path share the same channel. In contrast, conventional multihop networks suffer from adjacent node interference unless it is avoided with channel orthogonalization (Figure 2). Therefore, MIMO two-way relaying is more spectrally efficient compared to conventional SISO multihop networks.

To cancel adjacent node interference and multiplex forward and backward streams along the path, both linear algorithms (ZF or MMSE) and nonlinear MIMO algorithms (SIC/DPC) can be used [3]. In this paper, we only consider the linear algorithm where each TX or RX node, equipped with $M$ antennas, can cancel $M-1$ interference signals. Moreover, we consider the case $M=3$. At each node, there are two weights for the forward and backward paths. The adjacent interference signals at node $i$ are cancelled sequentially by a combination of its receive weights $\boldsymbol{w}_{i}^{r \mathrm{~F}}$ and $\boldsymbol{w}_{i}^{r \mathrm{~B}}$, and the transmit weigths $\boldsymbol{w}_{i+1}^{t \mathrm{~F}}$ and $\boldsymbol{w}_{i+1}^{t \mathrm{~F}}$ of its following node as illustrated in Figure 1. In the figure, $\perp$ means the weight is orthogonal to the effective channel of the interference signal, thus canceling it; and II denotes the weight is parallel to the direction of the desired signal. These antenna weights are calculated successively from left to right based on the knowledge of the left adjacent link channel information only. For more details on the MIMO twoway relaying algorithms, we refer the interested reader to [3].

In the following, we consider a wireless multihop network with $N$ nodes and $L=N-1$ active links. Because of the two-way transmission where RX and TX nodes switch their role at each time slot, we should distinguish between the sets of forward links and those of backward links in odd numbered time slots as $I_{\mathrm{o}}=I_{\mathrm{o}}^{\mathrm{F}} \cup I_{\mathrm{o}}^{\mathrm{B}}$, and in even numbered time slots as $I_{\mathrm{e}}=I_{\mathrm{e}}^{\mathrm{F}} \cup I_{e}^{\mathrm{B}}$. For odd time slots, the set of forward and backward links are $I_{o}^{\mathrm{F}}=\{1,3,5, \cdots\}$ and $I_{o}^{\mathrm{B}}=\{2,4,6, \cdots\}$ respectively. For even time slots, they become $I_{\mathrm{e}}^{\mathrm{F}}=\{2,4,6, \cdots\}$ and $I_{\mathrm{e}}^{\mathrm{B}}=\{1,3,5, \cdots\}$.

Next, we define the average effective channel gain between the TX node $T(k)$ of link $k$ and the RX node $R(l)$ of link $l$ by:

$$
g_{l, k}=\mathbf{E}\left[\left|\left(\boldsymbol{w}_{l}^{r, \operatorname{dir}(l)}\right)^{H} \widetilde{\boldsymbol{H}}_{R(l), T(k)} \boldsymbol{w}_{k}^{t, \operatorname{dir}(k)}\right|^{2}\right]
$$

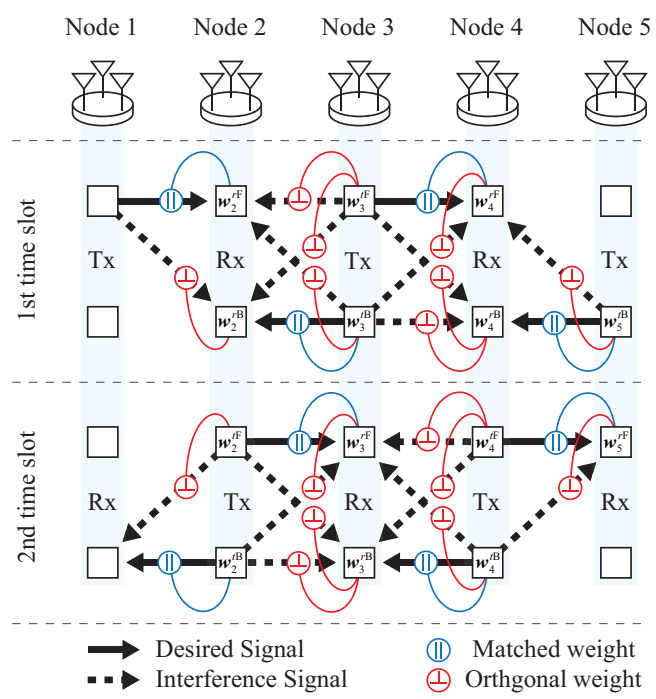

Figure 1. Model of MIMO two-way multihop network with linear processing.

where $\mathrm{E}()$ means expectation, the $\operatorname{dir}(l)$ and $\operatorname{dir}(k)$ indicates respectively the direction (forward or backward) of links $l$ and $k, \widetilde{H}_{R(l), T(k)} \in C^{M \times M}$ is the Rayleigh fading channel matrix between TX node $T(k)$ and RX node $R(l), \boldsymbol{w}_{j}^{t, \operatorname{dir}(l)} \in C^{M}$ is the transmit weight vectors of $T(k)$ for the indicated direction of link $k$ and $\boldsymbol{w}_{k}^{r, \operatorname{dir}(l)} \in C^{M}$ is the receive weight vector at $R(l)$ for the direction of link $l$.

\section{B. SINR expression}

The average signal to interference plus noise ratio (SINR) of link $l$ is obtained by

$$
\begin{aligned}
\gamma_{l} & =\frac{g_{l, l} P_{R, l}}{\sum_{k \neq l} g_{l, k} P_{R . l}+\sigma^{2}} \\
\gamma_{l} & =\frac{g_{l, l} D_{l, l}^{-\alpha} P_{l}}{\sum_{k \neq l} g_{l, k} D_{l, k}^{-\alpha} P_{k}+1}
\end{aligned}
$$

where we assumed a simplified path loss model $P_{\mathrm{R}, l}=$ $P_{\mathrm{T}, k} K\left(\frac{d_{0}}{D_{l, k}}\right)^{\alpha}$ and normalized the transmit power $P_{\mathrm{T}, k}$ over the noise power $\sigma^{2}$ as $P_{l}=\frac{P_{\mathrm{T}, k} K}{\sigma^{2}} \cdot K=\left(\frac{c}{4 \pi d_{0} f_{c}}\right)^{2}$ is the path loss at the reference distance $d_{0}=1 \mathrm{~m}, f_{c}$ is the center frequency, and $D_{l, k}$ is the distance between $T(k)$ and $R(l)$. The interference term in (2) comes only from far node interferers since all adjacent node interference signals are cancelled by the MIMO two-way linear scheme.

\section{Optimal Routing and Power Allocation}

In this section, we briefly review the cross-layer approach, based on the joint optimization of routing and power allocation, for mitigating the remaining interference from far nodes. The objective is to find the optimal relay locations as well as the optimal power allocation at each node that maximizes the bottleneck link SINR for both the forward and backward flows. In the following, we summarize the problem formulation which is based on the framework of geometric programming [9]. 


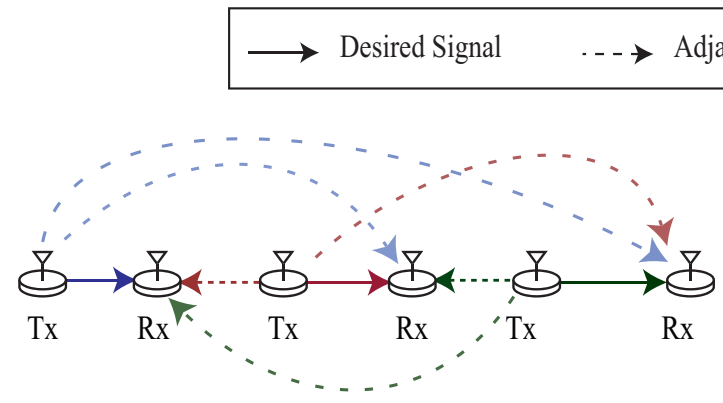

(a) Unidirectional transmission with adjacent node interference.
Adjacent Node Interference : :-.-A Far Node Interference

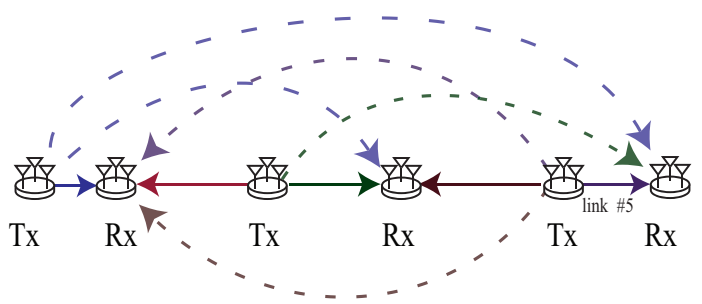

(b) Bidirectional transmission without adjacent node interference.

Figure 2. Comparison between: (a) conventional SISO multihop network; (b) MIMO two-way multihop network

Problem Formulation: Mathematically, the optimization variables are the node-to-node distance vector $\boldsymbol{d} \in \mathbf{R}^{N}$, and the power allocation vectors $\boldsymbol{P}_{o}, \boldsymbol{P}_{e} \in \mathbf{R}^{N}$ for odd and even time slots. We can consider two types of power constraints: per node power constraint and total power constraint. The latter will be used in the following analysis in order to allow a fair comparison of the performance of routing schemes with different number of hops. In each time slot, we constrained the multihop flow to allocate a maximum total power constraint $P_{\mathrm{T}}$ to its nodes as follows:

$$
\begin{aligned}
& \sum_{l=1}^{N} \boldsymbol{P}_{o}(l) \leq P_{\mathrm{T}} \quad \forall l \in I_{o} \\
& \sum_{k=1}^{N} \boldsymbol{P}_{e}(k) \leq P_{\mathrm{T}} \quad \forall k \in I_{e}
\end{aligned}
$$

so that the joint relay placement and power optimization problem is formulated by:

$$
\begin{array}{rr}
\text { variables } & \boldsymbol{d}, \boldsymbol{P}_{o}, \boldsymbol{P}_{e} \\
\text { maximize } & \min _{l \in I_{o} \cup I_{e}} \gamma_{l}
\end{array}
$$

subject to Total Power constraints in (3) - (4)

$$
\begin{aligned}
& \boldsymbol{d} \succ D_{\text {min }} \\
& \sum_{i=1}^{L} \boldsymbol{d}(i)=D_{\mathrm{E} 2 \mathrm{E}}
\end{aligned}
$$

where (6) is a minimum node-to-node distance constraint, and (7) is the end-to-end distance constraint.

By replacing $\gamma_{l}$ by (2) and introducing an auxilliary variable $t>0$, the optimization problem is equivalent to:

$$
\begin{array}{cc}
\text { minimize } & t \\
\text { subject to } & \frac{1}{\gamma_{l}}=\frac{\sum_{k \neq l} g_{l, k} D_{l, k}^{-\alpha} P_{k}+1}{g_{l, l} D_{l, l}^{-\alpha} P_{l}} \leq t \quad \forall l \in I_{o} \cup I_{e} \\
& \text { Constraints in (3), (4), (6) and (7) }
\end{array}
$$

where $D_{l, l}(\boldsymbol{d})=\boldsymbol{d}(l)$ is the distance of link $l$, and $D_{l, k}(\boldsymbol{d})$ is the distance between the RX node $R(l)$ of link $l$ and the TX node $T(k)$ of link $k$.

This optimization problem could be formulated as a generalized GP and was solved efficiently using a sequential GP method based on posynomial approximation called condensation technique [10]. We refer the reader to the technical report in [4] for more details. It can be proven that the solution $\boldsymbol{x}^{*}$ to

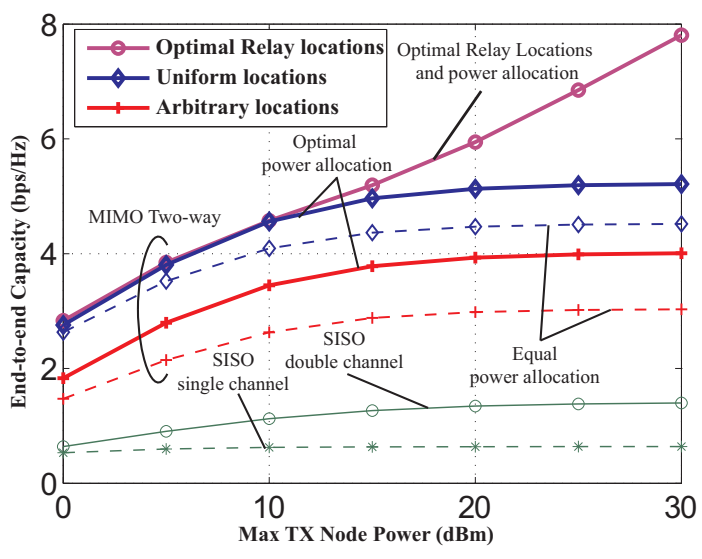

Figure 3. End-to-end capacity of different routing and power allocation strategies with $N=8$ nodes and $D_{E 2 E}=140 \mathrm{~m}$.

this sequential GP method always satisfies the Karush-KuhnTucker optimality conditions of the original problem [11].

\section{Performance of the intra-flow interference mitigation ap- proach}

Since the end-to-end performance of the multihop path is limited at the bottleneck link, we used as a figure of merit the average end-to-end capacity defined by:

$$
C_{\mathrm{E} 2 \mathrm{E}}=\frac{1}{2}\left[\mathbf{E}\left(C_{\mathrm{E} 2 \mathrm{E}}^{\mathrm{F}}\right)+\mathbf{E}\left(C_{\mathrm{E} 2 \mathrm{E}}^{\mathrm{B}}\right)\right] \quad[\mathrm{bit} / \mathrm{s} / \mathrm{Hz}]
$$

where $C_{\mathrm{E} 2 \mathrm{E}}^{\mathrm{F}}=\min _{l \in I_{o}^{\mathrm{F}} \cup I_{e}^{\mathrm{F}}} C_{l}$ and $C_{\mathrm{E} 2 \mathrm{E}}^{\mathrm{B}}=\min _{k \in I_{o}^{\mathrm{B}} \cup I_{e}^{\mathrm{B}}} C_{k}$ are the end-to-end capacity of forward and backward paths and $C_{l}=\log _{2}\left(1+\gamma_{l}(n)\right)$ is the capacity of link $l$ at time slot $n$.

Here, we briefly describe the achievable end-to-end capacity when only a single flow exists. As illustrated in Figure 3, we can see that routing has an important impact on the effectiveness of power allocation to mitigate intra-flow interference in multihop networks. Although all adjacent node interference are cancelled by the MIMO two-way relaying technique, the interference from far nodes can saturate the end-to-end capacity at high SNR. In fact, a poor choice of route, such as the uniform relay locations, resulted in large performance loss even with optimal power allocation. However, the jointly optimal routing and power allocation approach effectively reduced the effect of interference and achieved a significant capacity gain in interference-limited regime. Figure 3 also shows that our scheme offer much higher spectral efficiency than conventional SISO multihop networks. 
Single Hop Routing

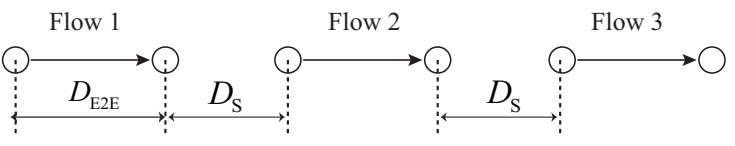

Multihop Routing

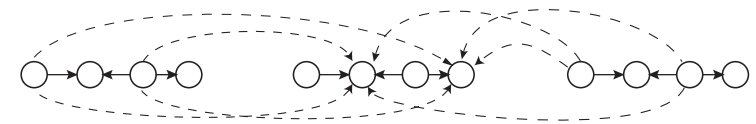

Inter-flow interference

Desired signal

Figure 4. Linear network topology with three flows (single hop and multihop)

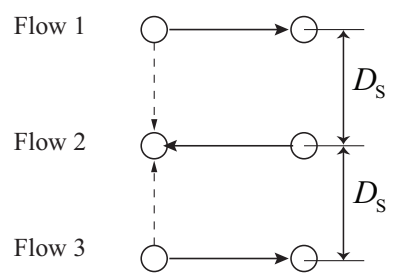

Single Hop Routing

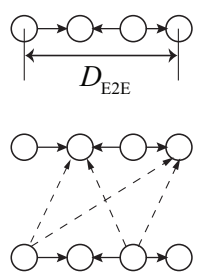

Multihop Routing
Inter-flow interference

Desired signal

Figure 5. Parallel network topology with three flows

\section{ANAlysis OF SPECTRAL EFFICIENCY WITH INTER-FLOW INTERFERENCE}

\section{A. Network Topology}

In the following, we consider a network with multiple single hop or multihop flows sharing the same channel and analyze the spectral efficiency when inter-flow interference is considered. We assume that the number of hops is the same for each flow but we vary the density of these simultaneous flows in two different classes of network topology: the linear topology (Figure 4) and the parallel topology (Figure 5). Although there are other topologies which do fit in these two types, the linear and parallel topologies are representative of the flow topologies encoutered in real networks. In both topologies, the flows are separated by a distance $D_{\mathrm{S}}$ and we change the ratio $R=\frac{D_{\mathrm{S}}}{D_{\mathrm{E} 2 \mathrm{E}}}$ of this separation distance and the end-to-end distance in order to control the density of the co-existing flows.

\section{B. Numerical Analysis}

We considered a network with three flows, which have the same end-to-end distance $D_{\mathrm{E} 2 \mathrm{E}}=140 \mathrm{~m}$. The simulation parameters are summarized in Table I.

In each topology, we measured the end-to-end capacity of the second flow since it was located in the middle of the three flows, thus receiving most of the inter-flow interference. Then, we compared the performance of the MIMO two-way technique with different number of hops with that of the single hop MIMO $3 \times 3$ transmission.

For the linear topology, we found that the end-to-end capacity of the single hop scheme vanished to zero when

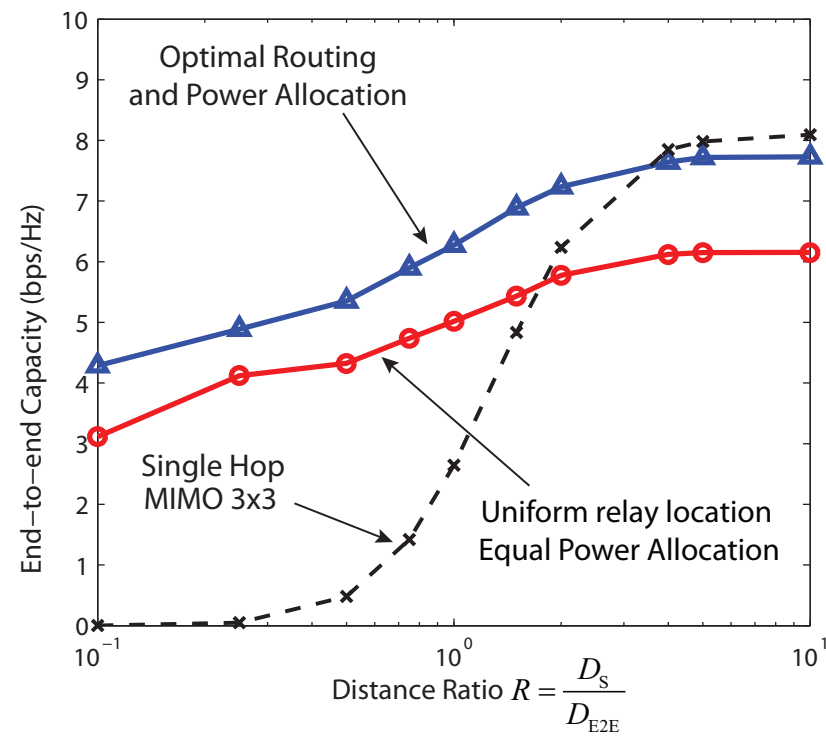

Figure 6. Effect of inter-flow interference on end-to-end capacity with an optimal number of hops and a total power constraint $P_{\mathrm{T}}=30 \mathrm{dBm}$

the density of the flows increases (Figure 7). On the other hand, the multihop routing could reduce the effect of interflow interference from nearby flows. With a total power constraint $P_{\mathrm{T}}=30 \mathrm{dBm}$, multihop $(N=4,6)$ and single hop routing provided almost the same capacity when there was no inter-flow interference (Figure 7). However, multihop routing outperformed the single hop transmission as the density of the flows increased, more precisely when $R<4$ in both topologies.

Figure 6 highlights this end-to-end capacity gain of multihop routing over single hop transmision in dense multihop flows. This gain is mainly due to the increase in number of hops, which reduces the transmit power per node, thus the inter-flow interference. Besides, an additional gain was obtained from the jointly optimal routing and power allocation, which also reduces the interference.

This means that MIMO two-way multihop routing is advantageous in terms of overall spectral efficiency in dense multihop networks. The only case where single hop is desirable, is when the SNR is very high (e.g. $P_{\mathrm{T}}=40 \mathrm{dBm}$ in Figure 7) and there is no co-existing flow in the neighborhood.

Our findings are similar to previous observations in [2], [7], [8] which assumed an orthogonal MAC scheme. The area spectral efficiency can be improved by multihop routing with reduced transmission power at the cost of the end-toend throughput. However, the MIMO two-way relay scheme combined with optimal routing and power allocation offers significantly higher spectral efficiency than conventional schemes.

Sikora et al. already showed in [8] that if full interference cancellation is performed, the spectral efficiency of multihop routing with spatial channel reuse can benefit from an increased number of hops. Instead of assuming a full interference cancellation such as in [8], however, we analyzed a more practical scheme with partial interference cancellation. Our scheme eliminates all intra-flow interference from adjacent node interference and reduces the remaining interference with optimal routing and power allocation. 

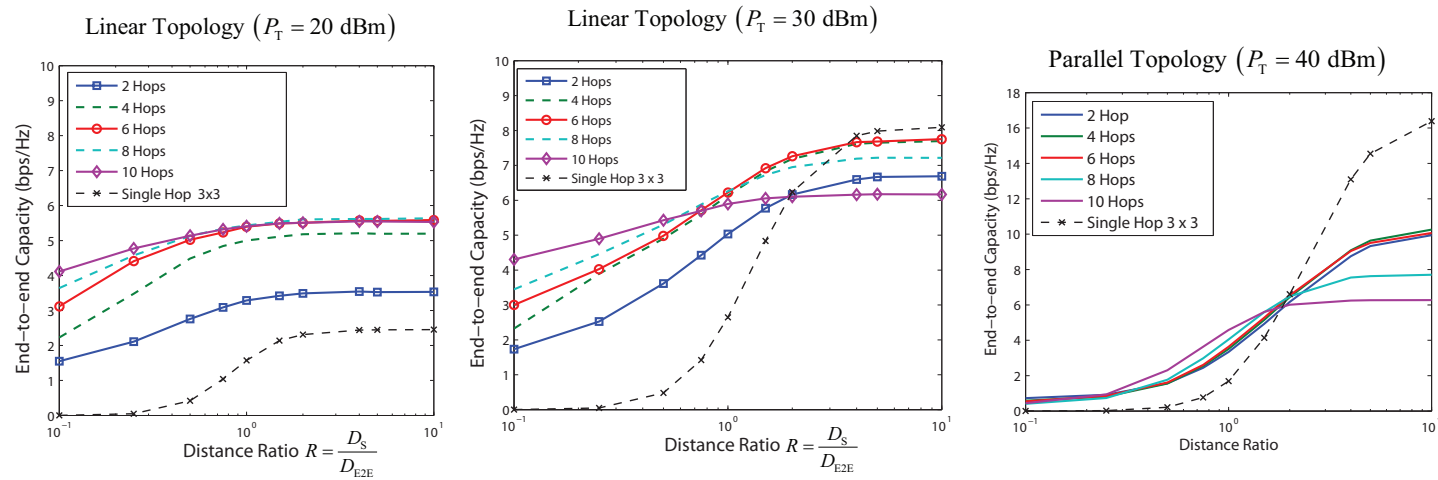

Figure 7. End-to-end capacity of flow no. 2 with different total power constraints in the in Linear Topology .

Finally, it can be noted from Figure 8 that the parallel topology is more prone to inter-flow interference. In fact, the flows almost overlap with each other when the separation distance is close to zero. It is not the case for the linear topology. For more general topologies, coordination among the nodes of the flows is needed to alleviate the inter-flow interference or to guarantee a QoS for each active flow. This can be done through distributed admission control and spectrum/power allocation schemes by imposing QoS and "interference-temperature" constraints [12], or by exchanging "interference prices" among the flows [13].

\section{Conclusion And Future Work}

We have studied the spectral efficiency of the MIMO two-way multihop networks when multiple flows co-exist in the network and share the same channel. In our approach to mitigate intra-flow interference, spatial array processing eliminates interference from adjacent nodes in a multihop flow while an optimal routing and power allocation strategy mitigates the remaining interference from far nodes. Our results showed that this approach also improved the overall spectral efficiency with co-existing flows in dense multihop networks, especially in the linear topology. It was due to the per node power reduction achieved by the multihop routing and by the power allocation. In the parallel topology, however, the strong inter-flow interference limited the spectrum sharing between multiple flows. Finally, when there was no other flow nearby, single hop MIMO transmission was desirable at high SNR regime because of higher spatial multiplexing gain.

Although these results are similar to observations in previous work, our approach for mitigating intra-flow interference is different and achieves higher spectral efficiency than conventional schemes. Our future work is to investigate distributed routing, flow admission control and channel allocation schemes for multihop networks with general topologies.

\section{ACKNOWLEDGEMENT}

The authors would like to thank Dr. Gia Khanh Tran at Tokyo Institute of Technology for his helpful discussions and comments.

\section{REFERENCES}

[1] IEEE 802.15 WPAN Task Group 4g (TG4g) Smart Utility Networks.

[2] P. Gupta and P.R. Kumar, "The Capacity of Wireless Networks," IEEE Trans Inf. Theory, vol. 46, no.2, pp. 388-404, Mar.
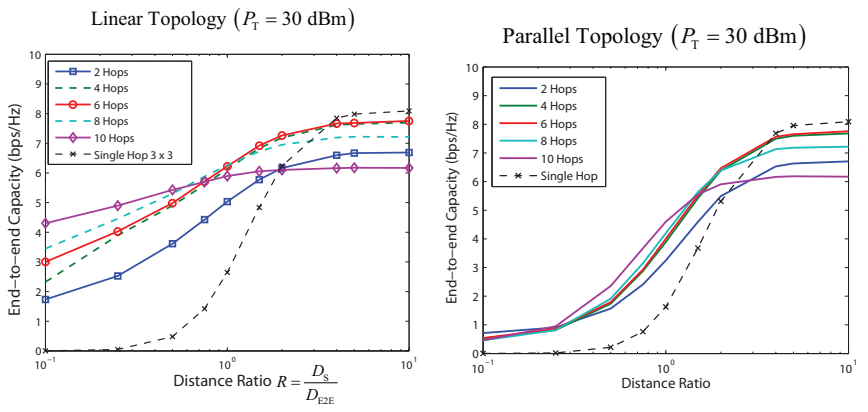

Figure 8. Effect of inter-flow interference in the linear and parallel topologies with a total power constraint $P_{T}=30 \mathrm{dBm}$

Table I

SiMULATION PARAMETERS

\begin{tabular}{|l|r|}
\hline Parameters & Values \\
\hline \hline Path loss exponent & 3.5 \\
\hline Center frequency & $2.4 \mathrm{GHz}$ \\
\hline Max TX node power & $0-30 \mathrm{dBm}$ \\
\hline Noise power & $-95 \mathrm{~m}$ \\
\hline Minimum node-to-node Distance & $2 \mathrm{~m}$ \\
\hline
\end{tabular}

[3] F. Ono and K. Sakaguchi, "MIMO spatial spectrum sharing for high efficiency mesh network," IEICE Trans. Commun., vol. E91-B, pp. 6269, Jan. 2008.2000

[4] R. Ramamonjison, J. Lee, K. Sakaguchi, and K. Araki. "Optimal Node Placement and Power Allocation in MIMO Two-Way Multihop Networks," IEICE Technical Report, RCS2009-285 pp.155-160

[5] Sikora, M.; Laneman, J. N.; Haenggi, M.; Costello, D. J. \& Fuja, T. "On the optimum number of hops in linear wireless networks," in Proc. IEEE Information Theory Workshop (ITW04), 2004, 165-169

[6] Florea, A., Yanikomeroglu. H. "On the optimal number of hops in infrastructure-based fixed relay networks," Global Telecommunications Conference, 2005. GLOBECOM '05. IEEE, 2005, 6, pp. 32-47

[7] Yamamoto, K. \& Yoshida, S. "Tradeoff between Area Spectral Efficiency and End-to-End Throughput in Rate-Adaptive Multihop Radio Networks," IEICE Transactions, 2005, 88-B, 3532-3540

[8] Sikora, M.; Laneman, J. N.; Haenggi, M.; Costello, D. J. \& Fuja, T. E. "Bandwidth- and power-efficient routing in linear wireless networks," IEEE Trans. Inf. Theory, 2006, 52, 2624-2633

[9] S. Boyd, S.-J. Kim, L. Vandenberghe, and A. Hassibi "A tutorial on geometric programming," Springer Netherlands, 2008.

[10] M. Avriel, "Advances in geometric programming," Plenum Press, 1980.

[11] B.R. Marks and G.P. Wright, "A General Inner Approximation Algorithm for Nonconvex Mathematical Programs," Operations Research, vol. 26, no. 4, pp.681-683, 1978.

[12] FCC Spectrum Policy Task Force, "Report of the spectrum efficiency working group," Nov. 2002. [Online]. Available: http://www.fcc.gov/sptf/files/SEWGFinalReport_1.pdf

[13] J. Huang, R. Berry, and M. L. Honig, "Distributed Interference Compensation for Wireless Networks", IEEE Journal on Selected Areas in Communications, Vol. 24, No. 5, pp. 1074-1084, May 2006. 